\title{
Reliability Analysis of an n-unit Standby Repairable System with K Repair Facilities
}

\author{
Xianyun Meng \\ College of Science, Yanshan University, Qinhuangdao 066004, China \\ E-mail: xiaoxia820@gmail.com \\ Hongxia Li \\ College of Science, Yanshan University, Qinhuangdao 066004, China \\ E-mail: xiaoxia209198282@163.com \\ Ning $\mathrm{Li}$ \\ College of Science, Yanshan University, Qinhuangdao 066004, China
}

\begin{abstract}
Redundancy or standby is a technique that has been widely applied to improving system reliability and availability in system design. In general, standby repairable system contains cold standby repairable system, hot standby repairable system and warm standby repairable system. In this paper, we shall deal with cold standby repairable system and warm standby repairable system with repair facilities. Under the assumptions that the failure time of unit and the repair time of failed unit are assumed to follow exponential distribution, we present a reliability analysis of an n-unit standby repairable system with $k\{k \in(1,2, \cdots, n)\}$ repair facilities.
\end{abstract}

Keywords: Standby repairable system, Reliability, Markov process theory, Steady-state quantities.

\section{Introduction}

Standby repairable system contains cold standby repairable system, hot Standby repairable system and warm standby repairable system. (Jinhua Cao,1986).has discussed the cold standby repairable system and the warm standby repairable system with only one repair facility, moreover, (Jianxiong Gu,2006) has discussed the cold standby repairable system with two repair facilities. In this paper, we shall deal with an n-unit standby repairable system with $k$ repair facilities. Under the assumptions that the failure time of unit and the repair time of failed unit are assumed to follow exponential distribution, we obtain the steady-state quantities and average quantities by using the Markov process theory.

\section{Cold standby repairable system}

\subsection{The assumptions of the model}

2.1.1 The system consists of $\mathrm{n}$ identical units and $k$ repair facilities. At the beginning $(t=0)$, all units are good, and one unit is working and others are under cold standby. The repair facility will repair the working one as soon as it fails. At the same time, the standby one begins to work. When the failed one has been repaired, it either begins to work again or becomes under cold standby. If the rest units fail while $k$ units are still under repair, they must wait for repair. The switch is perfect, instantaneous.

2.1.2 Assume that each unit after repair is 'as good as new'.

2.1.3 The failure time of unit and the repair time of failed unit are all exponentially distributed with parameter $\lambda$ and $\mu$ respectively. The failure time distribution and the repair time distribution are denoted which, respectively, by

$X \sim F(t)=1-e^{-\lambda t},(\lambda>0), t \geq 0 ; Y \sim G(t)=1-e^{-\mu t},(\mu>0), t \geq 0$,

The random variables are mutually independent.

2.2 The steady-state quantities and average quantities of the system.

Theorem 1: The system consists of $\mathrm{n}+1$ no-identical state. Let $x(t)=j,\{j=1,2, \cdots, n\}$ denote the state that there are $\mathrm{j}$ 
failed units (which contains the units that they are under repair) in the system at time $t$. So, the steady-state availability of the system is $A=1-\pi_{n}$; the steady-state failure frequency of the system is $M=\lambda \pi_{n-1}$; the mean up-time of the system is $M U T=\frac{A}{M}$; the mean down-time of the system is $M D T=\frac{1-A}{M} ;$ the mean cycle time of the system is $M C T=\frac{1}{M}$.

Where,

$\pi_{0}=\left\{1+\sum_{j=1}^{k} \frac{\lambda^{j}}{j ! \mu^{j}}+\frac{\lambda^{k}}{k ! \mu^{k}} \sum_{j=k+1}^{n}\left(\frac{\lambda}{k \mu}\right)^{j-k}\right\}^{-1}$

$\pi_{j}=\frac{\lambda^{j}}{j ! \mu^{j}} \pi_{0} \quad j=1,2, \cdots, k$.

$\pi_{j}=\frac{\lambda^{k}}{k ! \mu^{k}}\left(\frac{\lambda}{k \mu}\right)^{j-k} \pi_{0} \quad j=k+1 \cdots, n$.

The mean time to the first failure of the system is

MTTFF $=\sum_{i=0}^{n-1} x_{i}$

$$
\begin{aligned}
& =\frac{1}{\lambda}+f\left(\frac{1}{\lambda}\right)+f\left(f\left(\frac{1}{\lambda}\right)\right)+\cdots+\underbrace{f\left(f\left(\cdots f\left(\frac{1}{\lambda}\right)\right)\right)}_{n-k}+g_{k-1}(\underbrace{f\left(f\left(\cdots f\left(\frac{1}{\lambda}\right)\right)\right)}_{n-k}) \\
& +g_{k-2}\left(g_{k-1}(\underbrace{f\left(f\left(\cdots f\left(\frac{1}{\lambda}\right)\right)\right)}_{n-k})\right)+\cdots+g_{1}\left(g_{2}\left(\cdots g_{k-1}(\underbrace{f\left(f\left(\cdots f\left(\frac{1}{\lambda}\right)\right)\right)}_{n-k})\right)\right)
\end{aligned}
$$

Where,

$f(x)=\frac{1+k \mu x}{\lambda}$,

$g_{i}(x)=\frac{1+i \mu x}{\lambda}$

$i=k-1, k-2, \cdots, 1$.

Proof: By the definition of the cold standby repairable system, we can know that

$x(t)=j,\{j=1,2, \cdots, n\}$, The state $\mathrm{n}$ denotes that the system is down, and the rest states denote that the system is up.

Therefore $E=\{0,1, \cdots, n\}, W=\{0,1, \cdots, n-1\}, F=\{n\}$, so, the stochastic process $\{x(t), t \geq 0\}$ is Markov process with state space $E$.

The transition probability form different states in the time $\Delta t$ is equation (5).

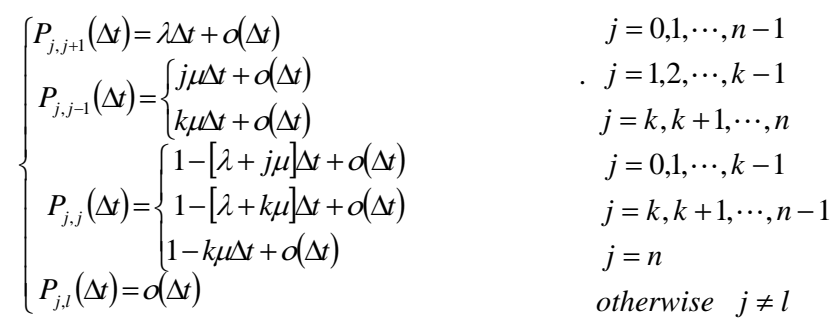

Where, $l \in(1,2, \cdots n)$.

And we get the matrix of transition probability that is matrix (6). 


$$
P=\left(\begin{array}{cccccc}
-\lambda & \lambda & & & & \\
\mu & -(\lambda+\mu) & \lambda & & & \\
\\
& 2 \mu & -(\lambda+2 \mu) & \lambda & & \\
\\
& \ddots & \ddots & \ddots & & \\
& & (k-1) \mu & -[\lambda+(k-1) \mu] & \lambda & \\
& & k \mu & -(\lambda+k \mu) & \lambda & \\
& & \ddots & \ddots & \ddots & \\
& & & k \mu & -(\lambda+k \mu) & \lambda \\
& & & & k \mu & -k \mu
\end{array}\right)_{(n+1) \times(n+1)}
$$

From the below equations (7), equation (4) can be obtained.

$$
\left(x_{0}, x_{1}, \cdots, x_{n-1}\right)\left(\begin{array}{cccccc}
-\lambda & \lambda & & & & \\
\mu & -(\lambda+\mu) & \lambda & & & \\
& 2 \mu & -(\lambda+2 \mu) & \lambda & & \\
& \ddots & \ddots & \ddots & & \\
& & (k-1) \mu & -[\lambda+(k-1) \mu] & \lambda & \\
& & & k \mu & -(\lambda+k \mu) & \lambda \\
& & & \ddots & \ddots & \ddots \\
& & & & k \mu & -(\lambda+k \mu)
\end{array}\right)=(-1,0, \cdots, 0)
$$

\section{Warm standby repairable system}

\subsection{The assumptions of the model}

3.1.1 The system consists of $\mathrm{n}$ identical units and $k$ repair facilities. At the beginning $(t=0)$, all units are good, and one unit is working and others are under warm standby. The repair facility will repair the working one as soon as it fails. At the same time, the warm standby one begins to work. When the failed one has been repaired, it either begins to work again or becomes under warm standby. If the rest units fail while $k$ units are still under repair, they must wait for repair The switch is perfect, instantaneous.

3.1.2 Assume that each unit after repair is 'as good as new'.

3.1.3 The lifetime distribution of all units is $F(t)=1-e^{-\lambda t}, t \geq 0,(\lambda>0)$, The lifetime distribution of standby units is $H(t)=1-e^{-v t}, t \geq 0,(v>0)$, the failure time distribution and the repair time distribution are $G(t)=1-e^{-\mu t}$, $t \geq 0,(\mu>0)$, The all random variables are mutually independent.

3.2 The steady-state quantities and average quantities of the system.

Theorem 2: The system consists of $\mathrm{n}+1$ no-identical state. Let $x(t)=j,\{j=1,2, \cdots, n\}$ denote the state that there are $\mathrm{j}$

failed units (which contains the units that they are under repair) in the system at time $t$. So, the steady-state availability of the system is $A=1-\pi_{n}$; is the steady-state failure frequency of the system is $M=\lambda \pi_{n-1}$; the mean up-time of the system is $M U T=\frac{A}{M}$; the mean down-time of the system is $M D T=\frac{1-A}{M} ;$ the mean cycle time of the system is $M C T=\frac{1}{M}$.

Where,

$$
\begin{aligned}
& \pi_{0}=\left\{1+\sum_{j=1}^{k} \frac{\prod_{i=1}^{j}[\lambda+(n-i) v]}{j ! \mu^{j}}+\frac{\prod_{i=1}^{k}[\lambda+(n-i) v]}{k ! \mu^{k}} \sum_{j=k+1}^{n} \frac{\prod_{l=1}^{j-k}[\lambda+(n-k-l) v]}{(k \mu)^{j-k}}\right\} \\
& \pi_{j}=\frac{\prod_{i=1}^{j}[\lambda+(n-i) v]}{j ! \mu^{j}} \pi_{0} \quad j=1,2, \cdots, k ;
\end{aligned}
$$


$\pi_{j}=\frac{\prod_{i=1}^{k}[\lambda+(n-i) v] \prod_{l=1}^{j-k}[\lambda+(n-k-l) v]}{k ! \mu^{k}} \pi_{0} \quad j=k+1, k+2, \cdots, n ;$

The mean time to first failure of the system is

$$
\begin{aligned}
\text { MTTFF }=\sum_{i=0}^{n-1} x_{i} & \\
= & \frac{1}{\lambda}+f_{1}\left(\frac{1}{\lambda}\right)+f_{2}\left(f_{1}\left(\frac{1}{\lambda}\right)\right)+\cdots+f_{n-k}\left(f_{n-k+1}\left(\cdots f_{1}\left(\frac{1}{\lambda}\right)\right)\right)+g_{k-1}\left(f_{n-k}\left(f_{n-k+1}\left(\cdots f_{1}\left(\frac{1}{\lambda}\right)\right)\right)\right) \\
& +g_{1}\left(\cdots+g_{k-1}\left(f_{n-k}\left(f_{n-k+1}\left(\cdots f_{1}\left(\frac{1}{\lambda}\right)\right)\right)\right)\right)
\end{aligned}
$$

Where,

$$
\begin{aligned}
& f_{i}(x)=\frac{1+k \mu x}{\lambda+i v}, \quad i=1,2, \cdots, n-k ; \\
& g_{j}(x)=\frac{1+j \mu x}{\lambda+(n-j) v}, \quad j=1, \cdots, k-1
\end{aligned}
$$

Proof: By the definition of the warm standby repairable system, we can know that $x(t)=j,\{j=1,2, \cdots, n\}$,

The state $\mathrm{n}$ denotes the system is down, and the rest states denote the system is up.

Therefore $E=\{0,1, \cdots, n\}, W=\{0,1, \cdots, n-1\}, F=\{n\}$, so, the stochastic process $\{x(t), t \geq 0\}$ is Markov process with state space $E$.

The transition probability form different states during the time $\Delta t$ is equation (12).

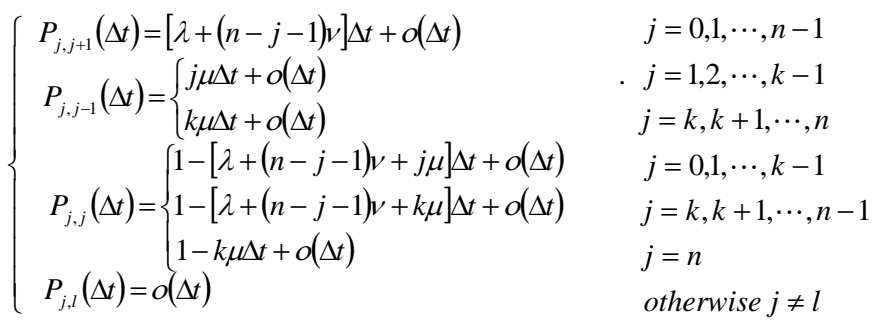

Where, $l \in(1,2, \cdots n)$.

And we get the matrix of transition probability that is matrix (13).

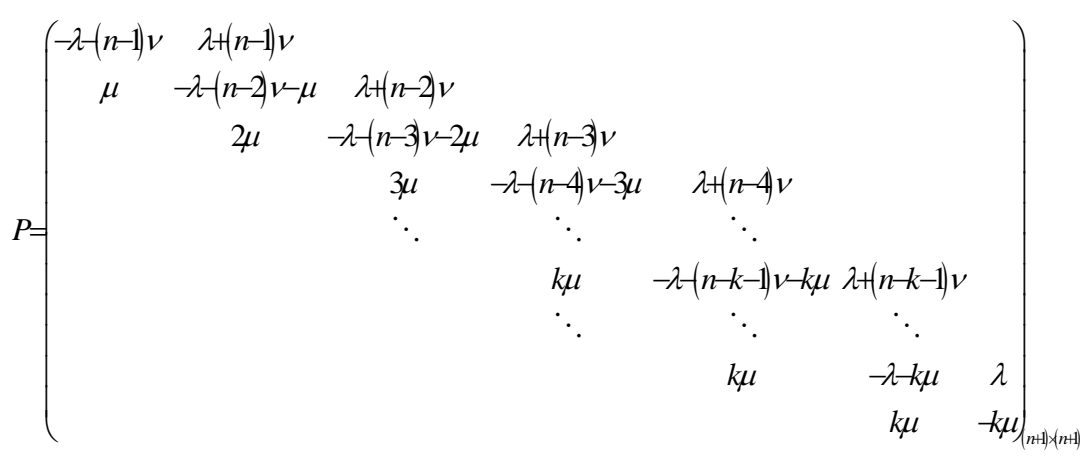

By calculating equations

$\left\{\begin{array}{l}\left(\pi_{0}, \pi_{1}, \cdots, \pi_{n}\right) P=(0,0, \cdots, 0) \\ \pi_{0}+\pi_{1}+\cdots+\pi_{n}=1\end{array}\right.$

and using matrix (13), we can get equations(8),(9)and(10).

From the above equations(14), equation (11) can be obtained. 


$$
\left(x_{0}, x_{1}, \cdots, x_{n-1}\right)\left(\begin{array}{cccccc}
-\lambda-(n-1) v & \lambda+(n-1) v & & & & \\
\mu & -\lambda-(n-2) v-\mu & \lambda+(n-2) v & & & \\
& 2 \mu & -\lambda-(n-3) \nu-2 \mu & \lambda+(n-3) v & & \\
& & 3 \mu & -\lambda-(n-4) v-3 \mu & \lambda+(n-4) v & \\
& & \ddots & \ddots & \ddots & \\
& & & k \mu & -\lambda-(n-k-1) v-k \mu & \lambda+(n-k-1) v \\
& & & \ddots & \ddots & \ddots \\
& & & & k \mu & -\lambda-k \mu
\end{array}\right)
$$

$=(-1,0, \cdots, 0)$

\section{References}

Cao, Jinhua \& Cheng, Kan. (1986). Introduction to Reliability Mathematics. Beijing: Science Press. pp. 183-238.

Chen, Guanjuan, Meng, Xianyun \& Liu, Yan etc. (2005). Reliability analysis of warm no maintained redundant of two different components repairable system with continuous lifetime switch.Journal of Yanshan University. pp. 408-413.

Gu, Jianxiong \& Wei, Yingyuan. (2006). Reliability Quantities of a n-unit Cold Standby Repairable System with Two Repair Facility. Journal of Gansu Lianhe University : Natural Sciences. pp. 17-20.

Meng, Xianyun, Liu, Yan \& Cheng, Guanjuan etc. (2006). Reliability analysis of warm standby repairable of two components with continuous lifetime switch and priority. Journal of Yanshan University. pp. 51-56. 